Accordingly, the state (national) veterinary service of Bosnia and Herzegovina $(\mathrm{BiH})$ conducted exercises which resulted in increased monitoring of wild and domestic bird populations and the drafting and adoption of a contingency plan (CP) for AI. The activities prescribed by the $\mathrm{CP}$ were implemented in February 2006 when the H5N1 virus was diagnosed in wild swans. However, no cooperation was established with public health authorities during this incident, further underscoring the need for a one health approach to disease control activities. Adoption of the One Health concept is challenging, and there is no simple plan that can be applied across all cultures. To prevent it from simply existing as an idealistic theory, some revision is needed and practical guidelines must be developed. The authors will include suggestions as to how this might be achieved.

Prehosp Disaster Med 2011;26(Suppl. 1):s51-s52

doi:10.1017/S1049023X11001798

(A184) Survey of Knowledge, Attitudes and Risk Perceptions (KAP) of Healthcare Personnel, in the Event of an Outbreak of H1N1 Influenza, in Limited Resource Environment

K. Aditya, ${ }^{1}$ A. Gadgil, ${ }^{2}$ J. Joseph, ${ }^{3}$ N. Roy ${ }^{4}$

1. Jamsetji Tata Centre for Disaster Management, Mumbai, India

2. Surgery, Mumbai, India,

3. Jamsetji Tata Centre for Disaster Management, 022, India

4. Public Health, Mumbai, India

Introduction: Little is known about the risk perceptions and attitudes of healthcare personnel, in the event of an epidemic of H1N1 influenza. It is acknowledged that perception of risks, as opposed to actual risks, alters behaviour. Indian data on KAP is needed as the scenario of working in limited resource environment, can have a different risk perception and attitudes amongst the healthcare workers. This will help in chalking out a 'model response planning' The study is designed to investigate the Knowledge and attitudes amongst healthcare workers in Mumbai, India during H1N1 pandemic of 2008-2009.

Methods: Questionnaire based interviews were given to healthcare workers, selected by their place of work, level of education and income group. This is based on the priori hypothesis that KAP differs amongst these groups. Surveys were distributed randomly to $20 \%$ employees from each of the above categories. Also healthcare officials, making policy decisions and guidelines, and the patients approaching these healthcare centres for treatment were interviewed for their perceptions of adequacy of the response measures. The questions designed included assessment of demographic characteristics, individual's knowledge about swine flu, perceived adequacy of training, perception of preparedness to tackle the epidemic situation and perception of risk to them and their families during epidemic conditions. The responses were graded as 'adequate or inadequate knowledge', perceived 'high or low risk' and 'tendency to apathy'. Results were analysed using statistical software (SPSS17).

Conclusion: Understanding the concerns and responses of healthcare personnel to a major infectious disease outbreak is critical to maintaining response capacity.

Prehosp Disaster Med 2011;26(Suppl. 1):s52

doi:10.1017/S1049023X11001804
(A185) Did the Ministry of Health's Intervention Increase Compliance of Medical Teams to be Vaccinated against $\mathrm{H} 1 \mathrm{~N} 1$ ?

B. Adini, ${ }^{1}$ D. Laor, ${ }^{2}$ T. Hornik-Luria, ${ }^{2}$ A. Goldberg, ${ }^{3}$

L. Aharonson-Daniel ${ }^{4}$

1. Emergency Division, Tel Aviv, Israel

2. Tel Aviv, Israel

3. Deputy Rector, Tel Aviv, Israel

4. Prepared Research Center, Tel Aviv, Israel

Background: Pandemic influenza poses a great challenge to healthcare systems. Vaccinating medical teams and the population against pandemic influenza is the global recommended strategy to contain spread of the disease. As part of the efforts made to overcome the H1N1 pandemic, the Israeli Ministry of Health $(\mathrm{MOH})$ initiated a general vaccination program for medical teams and the total country population. Due to low compliance rates of the medical staff, the $\mathrm{MOH}$ conducted regional conferences aimed at providing knowledge and encouraging staff to be vaccinated.

Objectives: To evaluate the effect of the regional conferences on the compliance rates amongst medical providers to be vaccinated against $\mathrm{H} 1 \mathrm{~N} 1$.

Methods: Medical providers from the primary health care services were invited to conferences that were conducted in 3 regions. Attitudes of the teams regarding compliance to be vaccinated were assessed pre and post the conferences. Additionally, the actual rates of vaccinations were recorded over the period of vaccination program. Actual compliance rates before and after the conferences were compared to detect differences as well as the relationship between teams' attitudes and actual vaccinations.

Results: Vaccination rates of medical providers remained low during the full vaccination period. Among the non-vaccinated, $24 \%$ to $29 \%$ reported before the conference that they agree to be vaccinated versus $57 \%$ to $62 \%$ following the conference. Analysis of the actual vaccination data among the medical providers did not demonstrate a change in compliance following the conferences and an overall decrease was noted after the first two weeks of the vaccinated project.

Conclusions: A statistically significant relationship was not found between reported attitudes of medical providers regarding readiness to be vaccinated and their actual vaccination. The $\mathrm{MOH}$ intervention did not achieve the expected result and did not raise compliance to be vaccinated.

Prehosp Disaster Med 2011;26(Suppl. 1):s52

doi:10.1017/S1049023X11001816

(A186) Edward via College of Osteopathic Medicine (VCOM) Honduras Dengue Outbreak Emergency Response Case Study

D. Sutphin, D. Tooke-rawlins, J. Willcox, J. Muller

International and Appalachian Outreach, Blacksburg, United States of America

In July 2010, the government of Honduras requested VCOM assistance with a widespread Dengue Outbreak. At the time of the mission trip, over 33,000 Hondurans had been hospitalized for Dengue Fever and a National State of Emergency declared. VCOM sent a team of medical students, faculty and volunteers to 
answer the call for help. The Honduran government selected five villages where the need for medical attention was greatest. The team provided medical exams, treatment or referral when necessary to approximately 200 patients in each of the five villages. General medical exams were provided in addition to screenings for Dengue Fever and subsequent supportive treatment including oral rehydration salts, Tylenol, vitamins and treatment of complications. National and local strategic partnerships to provide emergency medical response services included the Minister of Health, Minister of Defense, Minister of Transportation and Housing and office of the President of Honduras; Deans of the National and Catholic Medical Schools; President of the Board of Medicine and other governmental and non-governmental offices; Mayors and local officials, and often local media. At Marcala, the Minister of Public Transportation and Housing arrived by Helicopter from Tegucigalpa to greet the VCOM team and patients at the clinic; and at Santa Maria del Real, the Honduran President's son met the team personally. The Honduran support for VCOM's ongoing continuity of health and improved medical care efforts in the country was evident. The trip enhanced the skills and knowledge of participating students and faculty. Student Jenie Sales writes, "I not only enhanced my own clinical experience and knowledge, but I obtained a greater understanding for the people and culture of Honduras." Student opportunities for reflective learning included case study writing, surveys and evaluations. The successful experience will lead to increased knowledge in the care of patients during a Dengue outbreak.

Prehosp Disaster Med 2011;26(Suppl. 1):s52-s53

doi:10.1017/S1049023X11001828

\section{(A187) Red Cross Volunteers' Roles in Epidemic Control: Community-Level Interventions during Cholera Outbreaks in Zimbabwe and Haiti \\ P. Saaristo, T. Aloudat}

Health Department, Geneva 19, Switzerland

Managing epidemics, or preferably, preventing them, is a priority for the International Federation of the Red Cross (IFRC). The IFRC response to the cholera outbreaks in Zimbabwe in 2009 and in Haiti in 2010 both included: the Emergency Response Unit system as the backbone, and the International Red Cross Movement helped the National Red Cross Society fulfill its humanitarian mandate during the emergency. Water and Sanitation units and Basic Health Care Units cooperated seamlessly to ensure consistency and effectiveness in the activities. A large part of the International Red Cross and Red Crescent Movement response is performed by community-based volunteers. During both outbreaks, the Red Cross put special focus on community-level interventions. In both countries, the National Red Cross Society, supported by the International Federation of the Red Cross, trained volunteer groups using a local adaptation and translation of the IFRC training package for emergency health and epidemic control. Research has shown that community volunteers frequently lack the background information necessary for a quick and efficient response to epidemics, especially when they are located in areas that do not benefit from the support and guidance of health professionals. This is particularly true in developing countries that often lack sufficient healthcare facilities and staff. To help fill those gaps, the IFRC launched a training package Epidemic Control for Volunteers - more effectively involving volunteers in the epidemic management. It provides volunteers with a basic understanding of the diseases that can easily turn into epidemics. This training package is intended for volunteers and trainers in local branches of Red Cross and Red Crescent societies. It teaches them how they can help limit the number of victims, act quickly and effectively, and define their role in the community before, during, and after an epidemic.

Prehosp Disaster Med 2011;26(Suppl. 1):s53

doi:10.1017/S1049023X1100183X

(A188) Foot and Mouth Disease Continuity of Business Planning for the U.S. Dairy Industry

P.J. Hullinger, ${ }^{1}$ D. Bickett-Weddle, ${ }^{2}$ T. Goldsmith, ${ }^{3}$

J. Roth, ${ }^{2}$ J. Zack ${ }^{4}$

1. Veterinary Medicine and Epidemiology, Davis, United States of America

2. College of Veterinary Medicine, Ames, United States of America

3. College of Veterinary Medicine, Minneapolis, United States of America

4. Emergency Management and Diagnostics, Riverdale, United States of America

If foot-and-mouth disease (FMD) was detected in the United States (U.S.), a national animal health emergency would be declared and livestock and allied industries would feel the immediate impacts of animal and product movement restrictions, animal quarantines, disease surveillance activities and other necessary measures implemented to control the disease. These control measures, while necessary to contain the outbreak, would have impacts on the normal business practices of uninfected livestock producers in affected regions, and potentially disrupt interstate commerce. Such impacts would be most disruptive to industries producing perishable products and utilizing 'just-in-time' supply models. One significantly impacted sector would be the U.S. dairy industry whose operations rely upon daily animal, product and other supportive movements, and do not have the capacity to store milk for more than 24-48 hours. Disruption of normal milk movement in the U.S. could affect the provision of milk and milk products, as well as create significant milk disposal, environmental and animal welfare issues. The challenge of controlling and eliminating FMD while at the same time maintaining the long term viability of the U.S. dairy industry, represents a complex and multifaceted challenge. The United States Department of Agriculture (USDA) is collaborating in preparedness initiatives and pre-event, academia-facilitated emergency management planning efforts with states and livestock industries. A key element, critical to a successful outcome from this initiative is the involvement of industry throughout the process. One such effort is the 'Secure Milk Supply' (SMS) Plan project and its initial goal is to develop agreed upon processes and procedures to pick up, transport, and pasteurize milk from uninfected farms in FMD control areas thus helping to maintain business continuity for dairy producers, haulers, and processors. This presentation will describe the current approach to FMD control in the U.S., issues of special relevance to the dairy industry and the progress and planned future directions of the USDA sponsored SMS Plan. Prehosp Disaster Med 2011;26(Suppl. 1):s53 doi:10.1017/S1049023X11001841 